\title{
Instrument for Label-Free Detection of Noncoding RNAs
}

\author{
Peter Noy, ${ }^{1}$ Roger Steiner, ${ }^{1}$ Joerg Voelkle, ${ }^{1}$ Martin Hegner, ${ }^{2}$ and Christof Fattinger ${ }^{1}$ \\ ${ }^{1}$ F. Hoffmann-La Roche Ltd., Pharma Research and Early Development, Discovery Technologies, 4070 Basel, Switzerland \\ ${ }^{2}$ CRANN-The Naughton Institute, School of Physics, Trinity College Dublin, Dublin 2, Ireland
}

Correspondence should be addressed to Peter Noy, peter.noy@roche.com

Received 15 June 2011; Accepted 11 August 2011

Academic Editor: Maria Tenje

Copyright (C) 2012 Peter Noy et al. This is an open access article distributed under the Creative Commons Attribution License, which permits unrestricted use, distribution, and reproduction in any medium, provided the original work is properly cited.

\begin{abstract}
We set up a label-free direct binding assay for the detection of noncoding RNAs. The assay is based on nanomechanical cantilever arrays for the detection of surface stress induced by immobilized biomolecules and their interaction partners. We used various means to significantly reduce the drift of the cantilever readout that was a prominent feature in experiments with readout in stationary fluid before and after sample injection. Major improvements were achieved by focusing on a faster system equilibration (for instance temperature control and diffusion independence). Experimental protocols were improved to provide user-friendly and less time-consuming measurements. Further enhancements were achieved by, for example, using pre-gold-coated cantilever array wafers compared to individually prepared ones and a directly implemented data analysis tool as real-time feature of the measurement software. We have demonstrated picomolar specific biomarker target detection and can easily distinguish modified targets with single-nucleotide mismatches that hybridize with lower affinity.
\end{abstract}

\section{Introduction}

Nanomechanical sensing systems based on cantilever arrays are a basic research tool for exploring label-free assays. Investigators have shown several static mode applications for the detection of biological binding partners such as DNA hybridization [1-3] and receptor-ligand binding [4-7]. Our focus lies on the label-free detection of noncoding RNAs for medium throughput assays where half automated processes and less time-consuming protocols play an important role. Therefore our intention was to set up a stable and reliable device for this application in the field of genomics.

The detection of noncoding RNAs is of interest for monitoring miRNA or siRNA levels as biomarkers or for therapeutic approaches [8]. The present state of the art detection method for RNA is the branched DNA assay or DNA ELISA. As in an ELISA assay, an immobilized capture probe binds the target sequence. Afterwards the sandwich structure is completed with a detection probe (annotated as label extender). This label extender then binds the branched DNA with label probe. The labeled branches ensure a strong enough signal for detection [9]. The advantages of the DNA ELISA is that no amplification is necessary and no reverse transcription such as that in GPCR is needed. Measurements can be done directly on cell lysates. The fact that timeconsuming assay protocols are inherent for this ELISA type assay is a disadvantage. Furthermore, there is one major limitation: to attach the label we need a certain amount of nucleotides from the target strand which are not available for recognition and to ensure specificity.

For comparative measurement we refer to a publication where the label-free detection of biomarker transcripts in human RNA with a nanomechanical cantilever setup was shown [10].

As proof of concept for the newly designed setup our goal was to detect a single-stranded 21 mer oligonucleotide at $100 \mathrm{pM}$ in a physiological buffer solution.

For the detection of successful hybridization experiments we measured the transduced surface stress which accumulated depending on the amount of specifically bound ssDNA biomolecules. We operated our device in static mode and measured in liquid. The induced bending of the cantilever (which lies in the nanometer range) is measured by reflecting a laser beam on the top of the cantilever and pointing it towards a position sensitive detector (PSD) as described in $[11,12]$. Surface stress is induced by the interaction between immobilized biomolecules on the ssDNA biofunctionalized side of the cantilever bar and their interaction partners in 
an injected solution. Various forces such as intermolecular interactions, electrostatic forces, and changes in the electronic density of the cantilever surface lead to the resulting surface stress [13].

By subtracting the deflection signal of a nonspecific reference cantilever from the main signal, parasitic effects such as drift due to small temperature changes and nonspecific binding can be eliminated $[13,14]$.

Since measurable amount of signal drift is present in all known label-free detection methods we focused on its reduction by stabilizing the major external factors which affect drift in our nanomechanical setup. This was achieved by implementing a fast local temperature regulation system and measurement in continuous liquid flow. Our goal was to optimize the system towards semiautomatic device handling, which is essential for industrial applications.

To assist the interpretation of the recorded data we developed a real time analysis software which applies simple operations and plots the results concurrently with the measurement.

\section{Instrumentation, Materials, and Methods}

The cantilever deflection is measured by tracking a reflected laser spot on a position-sensitive detector (PSD) (1L10-10A SU15, SiTek Electro Optics, Sweden). As laser source we chose pigtail laser diodes of $635 \mathrm{~nm}$ wavelength (HL6320G, Opnext Japan Inc., Japan) and operated them in constant power mode. By arranging eight laser coupled fibers in a linear array we achieved readout of the eight cantilevers through their sequential illumination.

Our setup is divided into three parts. The main part is a temperature-controlled box containing the cantilever instrument and the fluidic system (Figure 1). To keep the temperature at the cantilever array stable, we installed two controlled loops. (i) An external flow cycle thermostat (ministat 125, Peter Huber Kaltemaschinenbau GmbH, Germany) to stabilize the temperature inside the temperaturecontrolled box. (ii) The second temperature regulation module is a Peltier element mounted inside the measurement chamber at a distance of about $2 \mathrm{~mm}$ from the cantilevers. The Peltier element was regulated by a Peltier controller which is normally used for laser temperature stabilization (LDT-5525, ILX LIGHTWAVE, USA).

We performed all measurements in liquid phase. Two syringe pumps (neMESYS system, Cetoni GmbH, Germany) pull the system liquid and samples through the measurement chamber. To compensate for the pressure loss due to pulling we applied 80 mbar (nitrogen) overpressure on all sample vessels and the system buffer reservoir. Halar tubing (Ercatech AG, Switzerland) was used to reduce loss of probe molecules in the sample due to adsorption onto the tubing surface.

For measurements the cantilever can be installed either under dry conditions or in a prefilled system where the chamber and tubing are filled with buffer. In both cases we flush the system with $\mathrm{CO}_{2}$ prior to filling with buffer. $\mathrm{CO}_{2}$

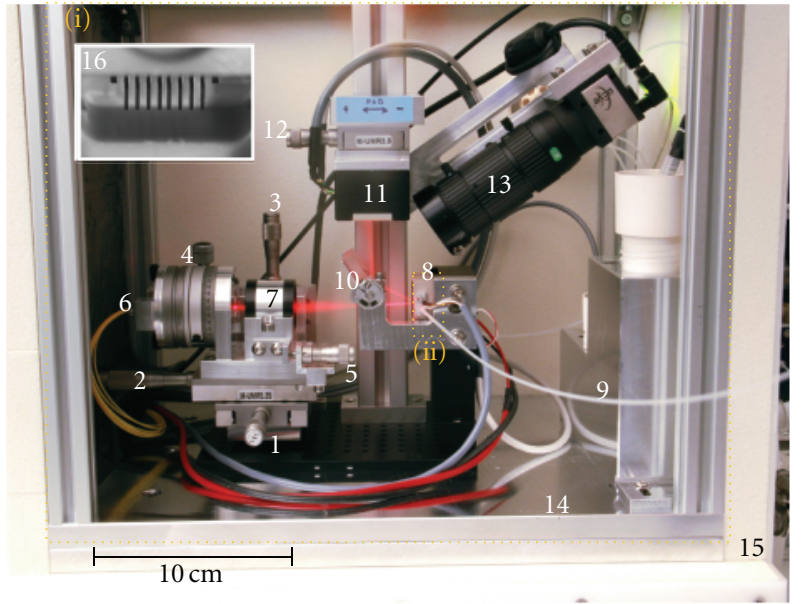

Figure 1: View inside temperature-controlled box containing the cantilever instrument. Laser ray path visible due to slight haze. (1, $2,3) \mathrm{x}, \mathrm{y}, \mathrm{z}$ positioning; (4) parallel alignment of fibers to cantilevers; (5) longitudinal focusing on cantilevers; (6) optical fibers (laser sources); (7) lens; (8) flow chamber (holds cantilever array chip); (9) tubing to syringe pump; (10) mirror with tilt function; (11) position-sensitive detector (PSD); (12) PSD alignment; (13) camera module; (14) ground plate connected to flow cycle thermostat; (15) thermal insulated box; (16) inset of cantilever array image mounted in flow chamber (8) taken with the camera module (13); (i) and (ii) illustrate the two temperature-controlled zones.

dissolves 80 times better in water than nitrogen and leads to a gas bubble-free fluidic system.

In addition to the temperature-controlled box the setup comprises a $19^{\prime \prime}$ rack containing the laser controller and power supply for the PSD.

The setup is controlled by LabView (NI PCI-6221 interface and LabView software kit, National Instruments, Switzerland). All measured values are recorded and processed by LabView software. The data analysis is based on algorithms which were tested and previously applied for kinetic microarray signals [15].

We used cantilever arrays with eight cantilever sensors precoated with $2 \mathrm{~nm}$ titanium $20 \mathrm{~nm}$ gold (IBM Research $\mathrm{GmbH}$, Switzerland). External dimensions of these sensors are as follows: $500 \mu \mathrm{m}$ length, $100 \mu \mathrm{m}$ width, and $0.5 \mu \mathrm{m}$ thickness. To regenerate and clean the gold surface of environmental organics for subsequent ssDNA functionalisation, the arrays were treated with UV ozone for 60 minutes (radiation flux at $185 \mathrm{~nm}: \sim 4 \mathrm{~W}$; ambient $\mathrm{O}_{2}$ ) prior to use [16]. An oxygen plasma treatment to clean the gold surface is not recommended due to the widely distributed electron energy leading to radiation damages and a poor controllability [16].

All measurements were performed under continuous flow $(10 \mu \mathrm{L} / \mathrm{min}$ for equilibration before and after the injections and $150 \mu \mathrm{L} / \mathrm{min}$ for the probe injection and wash step) using the above mentioned syringe pumps. Cantilever arrays were functionalized with thiol-modified ssDNA (Microsynth, Switzerland) for 60 minutes in acetic acid-triethylamine solution buffer in a home-built capillary device. Capillaries allow individual functionalisation of 


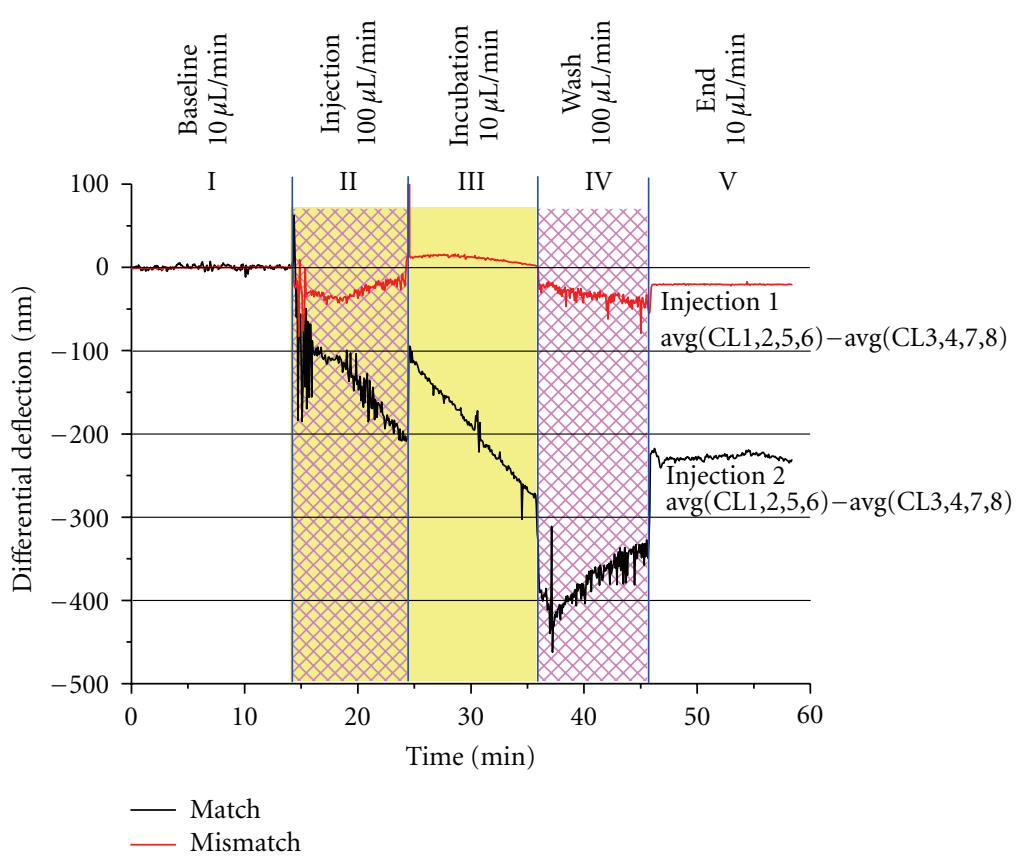

FIgure 2: Overlay of two consecutive experiments to prove the detection of a $100 \mathrm{pM}$ antisense strand. The graph shows the significant difference between an injection of $100 \mathrm{pM}$ antisense match strand (black curve) and an injection of $100 \mathrm{pM}$ antisense mismatch strand (red curve, the mismatch has two nonmatching base pairs in the centre of the target). Injecting the match sample induces approximately - $200 \mathrm{~nm}$ differential deflection, where else the injection of the mismatch configuration leads to almost no differential signal. Phase (I) shows the recorded baseline at $10 \mu \mathrm{L} / \mathrm{min}$ buffer flow. (II) $1,000 \mu \mathrm{L}$ sample injection at $100 \mu \mathrm{L} / \mathrm{min}$. (III) incubation phase at $10 \mu \mathrm{L} / \mathrm{min}$. (IV) flushing with buffer $100 \mu \mathrm{L} / \mathrm{min}$. (V) resulting differential deflection after injection cycle is completed $(10 \mu \mathrm{L} / \mathrm{min}$ buffer flow). Curves correspond to the differential deflection signal of positive minus reference cantilever (CL). Therefore the bending of the cantilevers is not absolute but differential deflections. The two injections were performed in series on the same cantilever array chip. A baseline correction, normalization, averaging, and differential signal calculation (probe minus reference) were done according to the literature [15]. Hatched area highlights the increased flow speed during injection and wash phase. Colored area indicates the presence of probe molecules in the flow chamber.

the various sensors. The DNA sequences chosen were AGAATAGGTATTTTTCCACAT for the biomarker target and AGAATAGGTATAATTCCACAT for the mismatch sequence. The chosen sequences do not tend to form hairpins and do not dimerize. In all experiments the following thiolated ssDNA oligonucleotides were used to functionalize the cantilever interface. (Sensor sequence: ATGTGGAAAAATACCTATTCT-C6 linker-SH, Reference sequence: CTTACGCTGAGTACTTTGA-C6 linker-SH). We used PBS (Invitrogen, Switzerland) as running and hybridization buffer.

\section{Results and Discussion}

With the described setup, we could detect a $100 \mathrm{pM}$ antisense strand and differentiate between a perfect match and mismatch sequence. Figure 2 shows the overlay of two consecutive experiments. Before each injection a stable baseline was recorded to ensure that all cantilevers were equilibrated (phase (I)). Due to the automated injection program the timing for the following injection steps was the same for each experiment. This allowed the overlay of the two sequential experiments shown in Figure 2. The effect of switching the valve from running buffer reservoir to the probe container and changing the flow speed from $10 \mu \mathrm{L} / \mathrm{min}$ to $100 \mu \mathrm{L} / \mathrm{min}$ is visible at the beginning of the sample injection in phase (II). It takes about 3 minutes until the sample reaches the chamber with the cantilevers. This explains why the slope did not change significantly until mid phase (II). The heavy fluctuations can be explained by the change in refractive index, flow effects, and the exchange of molecules in the chamber before a new equilibration is set. After $10 \mathrm{~min}$ the sample $(1,000 \mu \mathrm{L})$ is completely injected, the valve switches back to running buffer, and the flow speed is decreased to $10 \mu \mathrm{L} / \mathrm{min}$ (transition to phase (III)). In phase (III) the chamber is still filled with probe solution. A stable equilibrium is not reached during this incubation period. Several reactions leading to a cantilever deflection as described in [13] tend to occur. To remove the remaining probe solution and wash the chamber the buffer flow was increased (phase (IV)) to $100 \mu \mathrm{L} / \mathrm{min}$. We flushed with $1,000 \mu \mathrm{L}$ buffer. Here we see again that the delay before the probe solution in the system was fully replaced by buffer (change in slope). The peak at the changing point can be explained by the change in electrostatic conditions of the plain buffer solution compared to the buffer solution with probes. Fast effects such as valve switching and bulk buffer changes cannot be fully recorded due to the comparatively slow data acquisition $(0.25 \mathrm{~Hz})$, and therefore sequential injection traces are not completely identical. Finally the program switches back to the standby conditions 


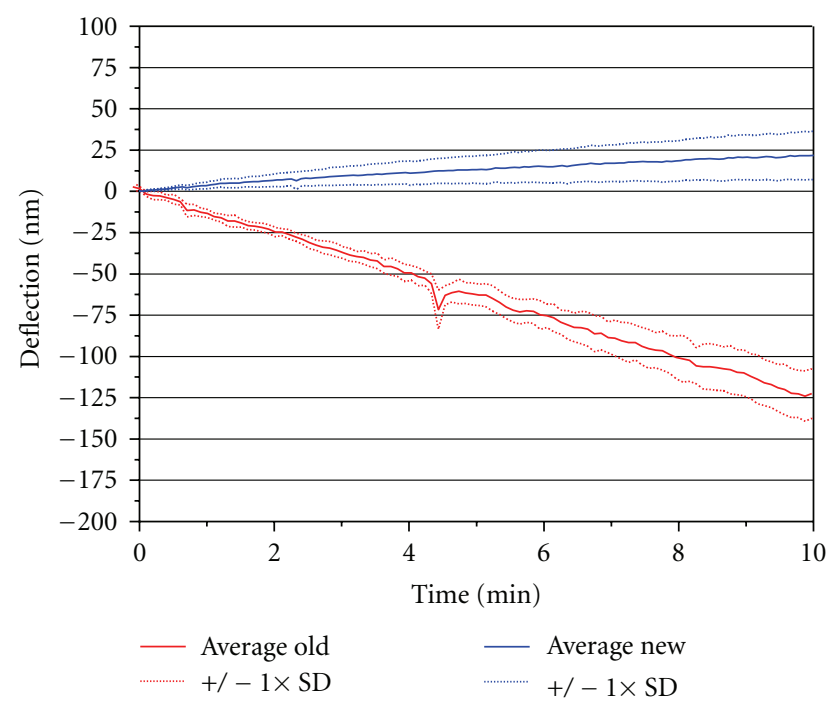

Figure 3: Typical drift before (red curve) and after implementation (blue curve) of means for drift reduction (continuous flow, temperature regulation, etc as described in this paper). Curves show the average of the raw data from 8 recorded cantilevers and the corresponding standard deviation. Curves have an offset at zero. Red curve measured under stationary conditions (flow: $0 \mu \mathrm{L} / \mathrm{min}$ ). Blue curve measured in flow $(10 \mu \mathrm{L} / \mathrm{min})$.

( $10 \mu \mathrm{L} / \mathrm{min}$ buffer flow), and the resulting deflection values are monitored. Compared to the end point of the deflection in phase (III), the start point of phase (V) is slightly higher $(\sim 50 \mathrm{~nm})$ although we have the same flow speed in phase (III) and (V): $10 \mu \mathrm{L} / \mathrm{min}$. A small amount of deflection is lost due to the dissolution of weakly bound strands (not fully hybridized) during the washing step. The two injections shown (red curve and black curve) were recorded sequentially. First, the negative probe (mismatch configuration) was injected and after a new equilibration the match injection was monitored. Finally the two starting points of the baselines were shifted to zero and the graphs plotted in an overlay. The resulting net deflection of $\sim 200 \mathrm{~nm}$ for the $100 \mathrm{pM}$ matching probe injection is repeatedly measured in our experiments. The resulting surface stress of about $9 \mathrm{mN} \mathrm{m}^{-1}$ is relatively high compared to previous experiments such as [10] (Young's modulus (Si): $130 \mathrm{GPa}$, Poisson ratio: 0.28). Reasons therefore could be due to longer cantilever functionalization times and due to different buffer properties which affect steric hindrance and ionic repulsion of the molecules.

By means of temperature stabilization and continuous flow measurements drift in the raw deflection signal was reduced from $\sim 12 \mathrm{~nm} / \mathrm{min}$ to $\sim 2.5 \mathrm{~nm} / \mathrm{min}$ as shown in Figure 3. The described setup and protocols represent a significant drift reduction by a factor 5 compared to previous experiments with readout in stationary fluid before and after sample injection. The gain in accuracy is especially of importance for the hybridization measurement with reaction times $>1$ min. The typical drift shown in Figure 3 was observed in all actual measurements.

In terms of electronic parts we used state of the art components. The amplifier has a noise level of approximately
$1 \mu \mathrm{V}$, the $\mathrm{PSD} \sim 3 \mu \mathrm{V}(\mathrm{BW}=100 \mathrm{~Hz})$. The analogue digital converter NI PCI-6221 with $\sim 122 \mu \mathrm{V}$ noise level is therefore the main source of electric disturbance (values from datasheet stated in $V_{\text {RMS }}$ to illustrate the critical components). Therefore we adjusted the full range scale to the maximum signal voltage and took the average over several measurement points (1,000 samples in $500 \mathrm{~ms})$. This is possible due to the slow reaction time $(>1 \mathrm{~Hz})$ compared with the sampling rate characteristics of the electronic parts. Furthermore we optimized the settling time of the laser controller and adjusted the data processing to let the laser stabilize after switching. Before averaging, we discard the first half of the data points to be sure to have a stable laser signal. The remaining 500 samples are still enough for noise reduction by averaging. Due to the sequential readout a too long sampling time might lead to missing a reaction event.

By placing a temperature-controlling element close to the cantilever array we obtained a controlled loop with very short time constant for temperature equilibration. Time to regulate the temperature in the chamber from $21^{\circ} \mathrm{C}$ room temperature to $25^{\circ} \mathrm{C}$ setpoint is approximately $0.5 \mathrm{~min}$. The much slower flow cycle thermostat regulation loop than that of the Peltier element leads to a stable temperature for all probe vessels, the buffer reservoir, and surrounding elements. In addition, a large $(23 \times 35 \times 2.5 \mathrm{~cm})$ aluminum ground plate provides a good heat exchange. To regulate the temperature from room temperature to setpoint by the flow cycle thermostat it takes $\sim 50 \mathrm{~min}$.

The two pulsation-free syringe pumps were embedded in our LabView control software. With two dosing modules an endless flow could be programmed, even for running measurements overnight. Besides electronic and temperature drifts the main portion of the overall drift visible in the deflection signal is drift due to diffusion effects (e.g., ionic exchanges between the cantilever surface and the surrounding liquid). The continuous flow led to a fast equilibration between the cantilever surface and the surrounding liquid which is diffusion independent. One feature which has to be taken into account when measuring in flow is the effect of the laminar flow on the cantilevers, as we see a deflection due to flow forces. In experiments with readout in stationary fluid before and after sample injection the liquid phase is moving during the injection process as well. This leads to significant flow induced deflections (see for instance in [10]). Depending on the position of the sensors relative to the liquid chamber channel the flow forces will be different for the eight cantilevers, inducing different additional bending that could potentially affect the measured deflection values. By measuring the baseline and the actual hybridization signal at equivalent buffer flow speeds, the comparability is given. The typical flow-induced bending by switching from stationary fluid to $10 \mu \mathrm{L} / \mathrm{min}$ is up to $7 \mathrm{~nm}$. For the increased injection flow rate the induced bending is up to $400 \mathrm{~nm}$ (see, e.g., Figure 2). Stop flow read out with only a short "stop" phase to record the data points (much smaller time period than the drift kinetics) could add additional improvement.

Due to instrument design restrictions (flow path, lack of space, and temperature sensibility) we decided to set 
up the flow with a syringe pump in pulling mode. The disadvantage with this pulling method is the risk of sucking air into the flow path. Small air bubbles will stick to the cantilever array and lead to an abortion of the measurement. By compensating the pressure loss with a positive pressure on the probe side we avoided these problems. Additionally, Halar tubing was chosen to avoid gas diffusion into the system. The gas permeability value for oxygen for Halar is similar to PEEK and $~ 30$ times less than Teflon (according to the specification guide from the provider). Moreover, Halar tubing is almost as flexible as Teflon tubing, in contrast to PEEK which would otherwise be a perfect material in terms of gas diffusion and low affinity for biomolecules.

Air bubbles tend to stick in small corners in the fluidic path and require a time-consuming procedure for their removal. $\mathrm{CO}_{2}$ sparging allows fast fluidic system priming without any bubbles. The buffering characteristics of the solution and closing the $\mathrm{CO}_{2}$ connection after priming ensure that the effect of the $\mathrm{CO}_{2}$ on the acidity of the buffer is negligible.

\section{Conclusion}

Equilibration time and drift were significantly reduced by the fast temperature control system and continuous flow measurement. After installing the cantilever chip, it takes about $1.5 \mathrm{~h}$ until the system is ready to measure. The major time-consuming step is the cantilever functionalization although the protocol was simplified by using pre-goldcoated arrays and $\mathrm{UV} / \mathrm{O}_{3}$ activation. With $\mathrm{CO}_{2}$ sparging, pressure compensation, and Halar tubing the formation of gas bubbles and their time-consuming removal was avoided. Further investigations into the effect of the continuous flow on the cantilevers will be carried out. The gain in drift reduction (approximately $10 \mathrm{~nm} / \mathrm{min}$ ) compared to the flowinduced bending $(\sim 7 \mathrm{~nm})$ leads to the assumption that a measurement under continuous flow is an improvement. State-of-the-art electronic components and investigations into signal stability led to a stable and reliable device (fluctuations $<5 \mathrm{~nm}$ for functionalized cantilever in liquid with a typical recording timescale of $0.25 \mathrm{~Hz}$ ). Device control, measurement, and data analysis by LabView lead to a fast and straightforward workflow. The specific detection of a short oligonucleotide strand at $100 \mathrm{pM}$ concentration in physiological buffer conditions demonstrated proof of concept of this setup.

\section{Acknowledgments}

We wish to thank Martin Hegner's group at the Trinity College in Dublin for guidance in setting up and testing the instrument and for providing valuable data for reference measurements. We acknowledge Ernst Meyer, University of Basel and Remo Hochstrasser, F. Hoffmann-La Roche, for their ideas and help for improvement of the instrumentation, Gregor Dernick, F. Hoffmann-La Roche, for his support in assay development, and Ulrich Certa, F. Hoffmann-La Roche, for support with the biological background.

\section{References}

[1] R. McKendry, J. Zhang, Y. Arntz et al., "Multiple labelfree biodetection and quantitative DNA-binding assays on a nanomechanical cantilever array," Proceedings of the National Academy of Sciences of the United States of America, vol. 99, no. 15, pp. 9783-9788, 2002.

[2] F. Huber, N. Backmann, W. Grange, M. Hegner, C. Gerber, and H. P. Lang, "Analyzing gene expression using combined nanomechanical cantilever sensors," Journal of Physics, vol. 61, no. 1, article 090, pp. 450-453, 2007.

[3] J. Mertens, C. Rogero, M. Calleja et al., "Label-free detection of DNA hybridization based on hydration-induced tension in nucleic acid films," Nature Nanotechnology, vol. 3, no. 5, pp. 301-307, 2008.

[4] Y. Arntz, J. D. Seelig, H. P. Lang et al., "Label-free protein assay based on a nanomechanical cantilever array," Nanotechnology, vol. 14, no. 1, pp. 86-90, 2003.

[5] T. Braun, M. K. Ghatkesar, N. Backmann et al., "Quantitative time-resolved measurement of membrane protein-ligand interactions using microcantilever array sensors," Nature Nanotechnology, vol. 4, no. 3, pp. 179-185, 2009.

[6] T. Braun, N. Backmann, M. Vögtli et al., "Conformational change of bacteriorhodopsin quantitatively monitored by microcantilever sensors," Biophysical Journal, vol. 90, no. 8, pp. 2970-2977, 2006.

[7] N. Backmann, C. Zahnd, F. Huber et al., "A label-free immunosensor array using single-chain antibody fragments," Proceedings of the National Academy of Sciences of the United States of America, vol. 102, no. 41, pp. 14587-14592, 2005.

[8] R. W. Carthew and E. J. Sontheimer, "Origins and Mechanisms of miRNAs and siRNAs," Cell, vol. 136, no. 4, pp. 642-655, 2009.

[9] M. L. Collins, B. Irvine, D. Tyner et al., "A branched DNA signal amplification assay for quantification of nucleic acid targets below 100 molecules/ml," Nucleic Acids Research, vol. 25, no. 15, pp. 2979-2984, 1997.

[10] J. Zhang, H. P. Lang, F. Huber et al., "Rapid and label-free nanomechanical detection of biomarker transcripts in human RNA,” Nature Nanotechnology, vol. 1, no. 3, pp. 214-220, 2006.

[11] H. P. Lang, R. Berger, C. Andreoli et al., "Sequential position readout from arrays of micromechanical cantilever sensors," Applied Physics Letters, vol. 72, no. 3, pp. 383-385, 1998.

[12] G. Meyer and N. M. Amer, "Simultaneous measurement of lateral and normal forces with an optical-beam-deflection atomic force microscope," Applied Physics Letters, vol. 57, no. 20, pp. 2089-2091, 1990.

[13] M. Godin, V. Tabard-Cossa, Y. Miyahara et al., "Cantileverbased sensing: the origin of surface stress and optimization strategies," Nanotechnology, vol. 21, no. 7, Article ID 075501, 2010.

[14] H. P. Lang, M. Hegner, E. Meyer, and C. Gerber, "Nanomechanics from atomic resolution to molecular recognition based on atomic force microscopy technology," Nanotechnology, vol. 13, no. 5, pp. R29-R36, 2002.

[15] T. Braun, F. Huber, M. K. Ghatkesar et al., "Processing of kinetic microarray signals," Sensors and Actuators, B, vol. 128, no. 1, pp. 75-82, 2007.

[16] W. Kern, Handbook of Semiconductor Wafer Cleaning Technology-Science, Technology, and Applications, Noyes Publications, 1993. 

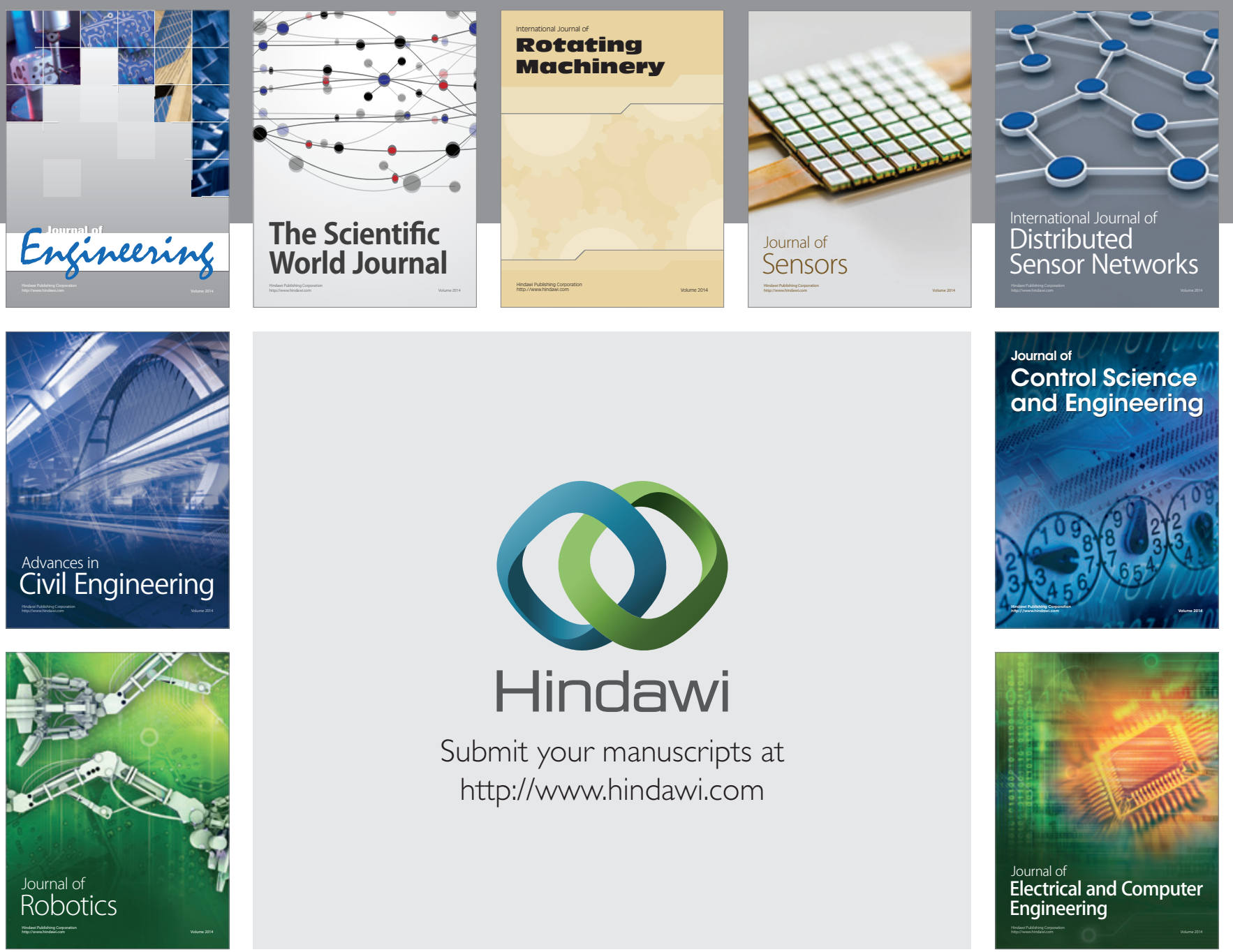

Submit your manuscripts at

http://www.hindawi.com
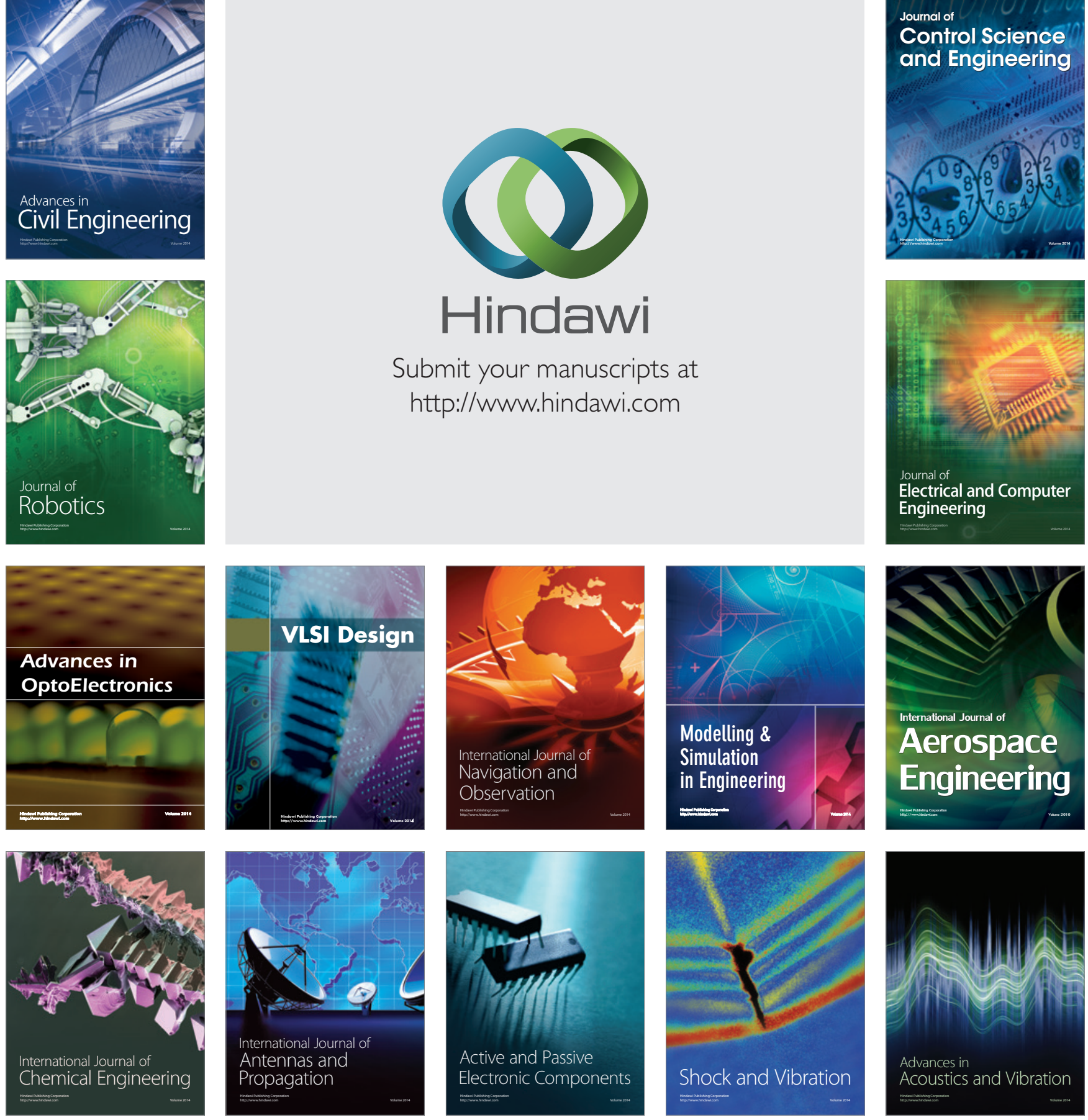